\title{
Response suppression as a function of the schedule of punishment'
}

DAVID S. CAMP, ${ }^{2}$ GEORGE A. RAYMOND AND RUSSELL M. CHURCH

BROWN UNIVERSITY

The schedule of punishment, like the schedule of positive reinforcement, is an important determinant of response rate. The amount of response suppression of rats in a free-responding situation was considerably greater for those given a variable-interval schedule of punishment than for those given a fixed-ratio schedule of punishment, even under conditions that roughly equated the frequency of punishments.

Although there is considerable information regarding the effects of various schedules of positive reinforcement on the behavior of animals, the effects of equivalent schedules of punishment are largely unknown. The one empirical generalization that is reasonably certain is that the magnitude of suppression is positively related to the rate of scheduled punishments. Thus, when punishment follows the next response after a fixed or variable interval of time the shorter the interval the slower the response rate; or, when punishment follows a fixed or variable number of responses, the smaller the ratio of responses to punishments the slower the response rate (Azrin, Holz, \& Hake, 1963)。

The present study attempts to compare the magnitude of response suppression produced by a punishment scheduled to follow a given number of responses (fixed ratio) with that produced by a punishment scheduled to follow the next response after a variable time period (variable interval)。Even under conditions that roughly equate the frequency of punishment, and with all other aspects of the situation constant (e.g., schedule of positive reinforcement, deprivation), there are gross differences in response suppression as a result of the schedule of punishment.

\section{Apparatus}

The apparatus consisted of a lever box with inside dimensions of $9-1 / 8$ in. $\times 8$ in., 8-5/8 in, high. The floor was composed of 16 stainless steel bars, $5 / 32$ in. diameter and spaced $9 / 16$ in. apart. A stainless steel lever $(1 / 2$ in. thick, 2 in. wide, and 2 in. above the floor) projected about $7 / 8$ in. into the box and it required a force of about $25 \mathrm{gm}$ to activate a microswitch. The box was enclosed in an ice chest which also contained a pellet dispenser, a $7-1 / 2-w$ bulb directly over the lever box, and a blower. Positive reinforcement was provided by $45-\mathrm{mg}$ rat food tablets (P. J. Noyes Co.). Punishment consisted of an electric shock delivered from a constant current stimulator (Applegate Model 250) through a scrambler (Lehigh Valley Electronics Scanner Model 1311) to the bars of the floor, the aluminum walls and the lever. The scramblers were located next to the lever box; the remaining control and recording apparatus was located in a separate room.

Subjects

The Ss were 24 naive, male, albino, Norway rats that arrived from the Charles River Breeding Laboratories at 60 days of age.

\section{Procedure}

Pretraining. The Ss were caged individually and water was accessible at all times in the home cages. Each day they were fed about
$14 \mathrm{gm}$ of ground Purina chow mixed with $25 \mathrm{cc}$ water. For the first five weeks Ss were handled once a day. On the first experimental day $S$ was placed in a lever box and one pellet was delivered each minute for $30 \mathrm{~min}$. On the second day of pretraining, $S$ was permitted to press the lever 30 times, and each response was reinforced. Thereafter all sessions were $30 \mathrm{~min}$. in length and each $\mathrm{S}$ was given its session at approximately the same time each day, five days per week.

Reinforcement training (Sessions 1-5). During the next five sessions Ss were reinforced on a 1-min. variable-interval schedule of positive reinforcement.

Punishment training (Sessions 6-15). 5). Twelve control Ss were given 10 additional sessions with 1 -min. variable-interval reinforcement and no punishment. The remaining $12 \mathrm{Ss}$ were randomly assigned to two groups of $6 \mathrm{Ss}$ each, both of which received a schedule of punishment in addition to the schedule of positive reinforcement. Under the conditions of "interval" punishment, a punishment was available on the average of every 2 min., so that an $S$ that continued to respond throughout the experimental session of $30 \mathrm{~min}$. received about 30 reinforcements and 15 punishments. Under the conditions of "ratio" punishment, a ratio was calculated for each $S$ such that, if its rate remained the same as that during the last session of reinforcement training it would receive 30 reinforcements and 15 punishments. All punishments were 25 ma intensity for $1.0 \mathrm{sec}$. duration.

Retraining (Sessions 16-22). Each $S$ was given 7 additional sessions of reinforcement training, i.e., 1-min. variable-interval schedule of positive reinforcement, and no punishment.

Measure

A "suppression ratio" was calculated for each $S$ on each of the sessions following regular training. This ratio was $B / A+B$, in which $A$ was the number of responses on the final day of regular training and $B$ was the number of responses on a particular day of punishment training or retraining.

\section{Results}

Figure 1 shows the degree of response suppression for Ss with the two schedules of punishment as a function of session. The mean suppression ratio during

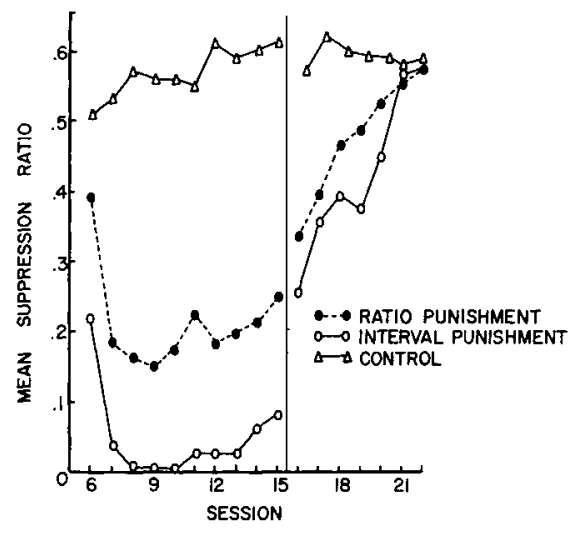

Fig. 1. The mean suppression ratio as a function of sessions of punishment training and retraining for groups with variableinterval and fixed-ratio schedules of punishment, and for an unpunished control group. 
the 10 sessions of punishment training under interval punishment, .049, was lower than under ratio punishment, $.211(\mathrm{U}=2, \mathrm{p}<.01)$. As a result of their greater resistance to the suppressive effect of punishment during the 10 sessions of punishment training, the ratio group received a mean of 25.7 reinforcements per session while the interval group received a mean of only 5.4 reinforcements per session $(U=1, p<.01)$. The two groups were similar with respect to the mean number of punishments received during the 10 sessions of punishment training $(4.4$ per session for the ratio group and 3.4 per session for the interval group, $U=12$, $p=.20$ ). During retraining each of the $S s$ in the ratio punishment group gradually increased its response rate; Ss in the interval punishment group abruptly returned to a response rate similar to the pre-punishment rate either immediately at the beginning of retraining or after a few additional sessions of nearly total response suppression. This is reflected in the significantly greater variability of suppression ratios of the first session of retraining by Ss in the interval punishment group than by $\mathrm{Ss}$ in the ratio punishment group (Moses Test of Extreme Reactions, $S=7, p<.05$ ). The two groups were indistinguishable on the last two days of retraining, and the apparent mean difference during the early sessions of retraining was not supported by statistical analysis. Although the punished Ss on the last session of retraining had a mean suppression ratio significantly above .500 (Wilcoxon $T=1, p<.001$ ), this is not evidence for a "compensatory rebound" from the effects of punishment since it was not significantly different from that of the unpunished control group during the last session of retraining $(U=55, p>010)$. Discussion

The present experiment demonstrates that the schedule of punishment is an important determinant of the amount of response suppression. The Ss in the ratio punishment group typically decreased their response rate considerably and spaced their responses so that they received virtually all of the reinforcements available but few of the punishments; Ss in the interval punishment group typically ceased responding. Because the two groups were equivalent with respect to the frequency of punishments actually received, the significant difference in the amount of suppression produced by the punishments was undoubtedly related to some other characteristics of the schedule on which they were administered.

Although this experimental variation in the schedule of punishment in a free-responding situation cannot isolate a responsible quantitative variable, it suggests several parameters of potential importance for the amount of suppression observed: (a) The amount of response suppression may be directly related to the probability that the response will be punished, since the percentage of responses that were punished was considerably greater in the interval group than in the ratio group. (b) If other variables were constant, the amount of response suppression may be inversely related to the probability that the response will be positively reinforced. (c) The magnitude of response suppression may be directly related to the proportion of the events (reinforcements and punishments) that are punishments. Thus, the interval punishment group may have been more suppressed than the ratio punishment group because the proportion of events that were punishments was substantially greater in the interval punishment group. (d) The consequence of a response that is not followed by either positive reinforcement or punishment is uncertain. The ratio $S$ is guaranteed either a number of these null trials or positive reinforcements following each punished response, and they may be responsible for some of the resistance to suppression observed in this group. (e) The momentary probability of each of the three consequences of a response (positive reinforcement, punishment, or null trial) may be more important than the overall probabilities. For example, the momentary probability of a punishment increases as a function of time in the interval group, so that as the inter-response time increases, the probability of a punishment approaches unity. (f) Finally, the magnitude of response suppression, and its variability, may be related to the correlation between response rate and punishment rate. In contrast to Ss in the interval group, $\mathrm{Ss}$ in the ratio group are in a negative feedback situation (producing differential punishment of fast rate of responding) since the punishment rate is directly related to the response rate.

The schedule of punishment, like the schedule of positive reinforcement, is an important determinant of response rate. Separate experiments are necessary to evaluate the relevance of various characteristics of schedules of punishment for the amount of response suppression.

\section{Reference}

Azrin, N. H., Holz, W. C., \& Hake, D. F. Fixed-ratio punishment. J. exp. Anal. Behav., 1963, 6, 141-148.

\section{Notes}

1. This investigation was supported by Public Health Service Research Grant MH 08123, from the National Institute of Health.

2. Now at the University of Rhode Island. 